\title{
Sistema automatizado para la gestión y control de requerimientos de soporte técnico
}

An automated system for requirements management and control technical support

Daniel Garófalo

garofalodaniel@gmail.com

Código ORCID: 0000-0001-6313-9382

Universidad Alejandro de Humboldt, Venezuela

Articulo recibido en enero 2019
$<\quad$ Arbitrado en febrero 2019
$<$ Publicado en julio 2019

Resumen

En algunas empresas, las actividades de control de incidencias de los requerimientos de los usuarios de equipos informáticos se llevaban en forma manual. Este método generaba retraso y lentitud en el registro, control y seguimiento de los incidentes, además del extravío de anotaciones debido a problemas de seguridad en el acceso a los datos, sin respaldo inmediato. En consecuencia, se diseñó para la empresa un sistema pionero capaz de registrar y controlar los diferentes requerimientos, ahorrar tiempo y realizar las tareas de manera rápida, sencilla, eficaz, además de proporcionar seguridad y respaldo. El trabajo se llevó a cabo bajo la modalidad de proyecto factible, con una muestra significativa y una aplicación exitosa en una empresa venezolana de distribución y manejo de géneros farmacéuticos. Se presenta el diseño y un ejemplo de generación de información en la historia de la creación de software. Se concluye con el cumplimiento cabal del propósito.

Palabras clave: Sistemas de control y registro, ambiente web, ingeniería informática, aplicación de software

\begin{abstract}
In some companies, the incident control activities of the users' computer equipment requirements were carried out manually. This method generated delay and slowness in the registration, control and monitoring of the incidents, in addition to the loss of annotations due to security problems in the access to the data, without immediate support. Consequently, a pioneer system designed to record and control the different requirements, save time and perform tasks quickly, easily, efficiently, as well as providing security and support, was designed for the company. The work was carried out under the feasible project modality, with a significant sample and a successful application in a Venezuelan pharmaceutical distribution and management company. The design and an example of information generation in the history of software creation are presented. It concludes with the full fulfillment of the purpose.
\end{abstract}

Keywords: Control and registration systems, web environment, computer engineering, software application 


\section{INTRODUCCIÓN}

La informática es una herramienta empleada en todo el mundo por toda clase de empresas. La tecnología actual y la informática han ayudado a acrecentar la eficiencia y eficacia de las industrias, ofreciendo seguridad y rapidez en el manejo de la información. Para desarrollar un sistema, es necesario el estudio metódico de toda la información recabada mediante la investigación y el análisis: levantamiento cabal de la información para ofrecer una solución confiable a los problemas encontrados, además de satisfacer las necesidades y requerimientos de los usuarios del sistema en desarrollo.

La administración consiste en la coordinación de todos los recursos a través de los procesos de planificación, ordenación, orientación y control en función de conseguir los objetivos establecidos (Stoner, Freeman y Gilbert, 1996). Es la actividad que, bien ejercida y con las herramientas apropiadas, es factor fundamental en la realización de cualquier empresa y que, además, es vital en las actividades de las industrias pequeñas y medianas.

La necesidad de proveer a las empresas emergentes de un sistema automatizado que registre $y$ controle $y$ actualice los requerimientos de soporte técnico a usuarios de sistemas informáticos ha ido creciendo desde hace décadas, debido a la dependencia de los equipos y software empleados que permiten el manejo de grandes volúmenes de información para el negocio de la empresa, y que crece cada día más (Howard, 2003). Se han necesitado sistemas que puedan llevar el registro, control y actualización de todos los requerimientos de soporte técnico efectuados por los equipos informáticos. Este sistema, en particular, fue aplicado para incrementar el rendimiento y la productividad del personal que labora en el área de Soporte Técnico, aumentando la seguridad de la información y respuesta rápida a las solicitudes de servicio, realizadas por los trabajadores de la empresa. En su momento, fue sumamente importante para el personal que se encontraba en el campo, poder a través de sus teléfonos -sin el proceso de efectuar una llamada- utilizar un vínculo web, colocar la incidencia, recibir la respuesta del servidor y que se registrara directamente en el módulo web en tiempo real, del lado del Administrador.

En el departamento de Sistemas de Información de la empresa vinculada con el mercado farmacéutico y de salud implicada en la propuesta, específicamente en el área de Análisis de Soporte, se llevaba a cabo todo el procesamiento de requerimientos de soporte técnico por parte de usuarios de equipos informáticos y el registro y manejo de base de conocimientos que las operaciones ligadas a estas actividades proveen. Dentro de las principales actividades del Analista de Soporte asignado a esta área están la ejecución de cualquier medida basada en las decisiones tomadas por la Gerencia de Sistemas y el procesamiento de reportes o requerimientos hechos por los usuarios finales de equipos informáticos; equipos que la empresa otorga para la realización de actividades laborales por parte del personal dentro y fuera de las instalaciones.

Debido al procesamiento en forma manual se presentaban fallas, errores o incongruencias en los datos utilizados para el registro y control de la información básica provista por el usuario al momento de la elaboración del reporte y en la información 
resultante del análisis, resolución y documentación de la falla. Este tipo de operaciones se gestionan a través de computadores, agendas pda, smartphones, sistemas de información y otros equipos electrónicos.

El reporte, registro y control de requerimientos de soporte técnico por parte de usuarios de equipos informáticos se llevaban a cabo de la siguiente manera: (a) si el usuario final percibía alguna falla $o$ desajuste en alguno de sus equipos informáticos asignados, procedía a reportar la dificultad a través de una llamada telefónica o a través de un correo enviado directamente al área de Análisis de Soporte, al analista de guardia; (b) si el reporte llegaba a través de una llamada telefónica se debía llenar el formato en Microsoft Excel con la fecha de realización de requerimiento, su identidad o nombre de usuario y una breve descripción de la falla; (c) si el reporte de la falla llegaba por correo electrónico se llenaba el formato con la información recibida en línea. Esto provocaba retrasos innecesarios en la realización de las actividades propias de cada una de las partes implicadas. De igual manera, no ofrecía un respaldo efectivo, moderno y ajustado a las altas demandas de operatividad en el tiempo, visto en los reclamos y quejas por parte de los usuarios finales por la poca velocidad y falta de precisión de aquellas tareas que deben ser llevadas a cabo por ambos implicados, sea el área de Análisis de Soporte o sea el usuario final.

Debe recordarse que la solución expedita en esos ámbitos es parte fundamental y crítica debido a la alta tasa de uso de sistemas computarizados. Una vez más, el acceso a documentación pública, estudios clínicos, Internet y telefonía está condicionado a la plataforma de red o intranet preestablecida en la empresa. Cualquier falla técnica, sea dentro del área de software, hardware o redes, implicaría un retraso en el desenvolvimiento de las actividades y operaciones del personal. Estas situaciones admiten un alto riesgo para el usuario final al no poder solventar o aclarar cualquier dificultad que esté presentando su plataforma tecnológica (Alcalde, 2001).

Los problemas se extienden desde la posible denegación de acceso a documentos públicos ubicados en la intranet de la empresa, falla del acceso telefónico para el personal que labora dentro y fuera de las instalaciones, posible pérdida de documentos personales o falla de acceso a la intranet e Internet, para el acceso a portales especializados de investigaciones clínicas y falla o demora en el reporte de visitas médicas por parte de los representantes de ventas.

Por otra parte, el Departamento de Sistemas de Información debe proveer servicios y soluciones de carácter tecnológico-informativo y acceso a documentación y redes (voz o datos) de manera confiable, continua y segura, por lo que requiere la constante documentación de fallas, contratiempos y fluctuaciones en la calidad de todos los servicios para los usuarios finales. Si no se tiene el sistema indicado sobrevendrían retrasos y complicaciones en la oferta de servicio y soporte, falla en el registro y documentación de los casos por atender y atendidos y fallas en el registro de información que pueda aumentar la base de conocimientos del área de soporte y que a su vez, suministre datos valiosos para solventar futuros inconvenientes a nivel técnico (Hamidian y 
Ospino, 2015). Otras desventajas del registro manual son el bajo nivel de seguridad para proteger los archivos informáticos y el nivel de acceso para restringir los cambios indeseados; la pérdida de los archivos de la base de conocimiento compilada, sin ningún respaldo físico actualizado y poca seguridad en el resguardo por la ausencia de claves de acceso a la información.

Este problema afectaba tanto a los usuarios finales, quienes debían trabajar con el uso de documentos (que puede ser de carácter médico, administrativo y de finanzas) y ofrecer soluciones en el área de negocios a todos los proveedores de servicios de laboratorio, como al personal médico asociado y otras sucursales de la empresa con quienes se intercambia información de todo tipo. Es un problema de envergadura, ya que todos los entes mencionados dependen de una administración eficiente y eficaz en el manejo de la documentación, información, servicios informáticos y electrónicos que son parte fundamental de la vida diaria, más aún en el área de negocios.

Por todas las razones expuestas, se propuso diseñar un sistema automatizado para el control, registro y actualización de requerimientos de soporte técnico efectuados por los usuarios de equipos informáticos. Con ello se analizaron previamente los registros, se establecieron la fundación y plan de elaboración arquitectónica del proyecto, se suministró una base para la eliminación de riesgos potenciales de carácter técnico mediante la construcción de una solución preliminar en forma de prototipo, se completó la funcionalidad del sistema mediante el estudio de los requisitos pendientes, administrando los cambios de acuerdo a las evaluaciones realizados por los usuarios y se aseguró que el software estuviera disponible para los usuarios finales, eliminando los errores y defectos encontrados en las pruebas de aceptación y asegurando la capacitación a los usuarios así como el soporte técnico.

Estévez (2008) afirma que este tipo de sistemas por su carácter de interactividad con el usuario sirve de soporte para cualquier tarea que tenga que ver con el adiestramiento del personal, solucionando las dificultades con la preparación de sus analistas. Asimismo, asume que la simplicidad y eficacia de este sistema lo dota de la suficiente flexibilidad para ser incorporado en otro sistema de información aún más complejo, como la incorporación de cursos u otros temas de interés con el que se vea beneficiado el personal.

También, Uzcátegui (2006) asevera que estos sistemas son eficientes en la disminución del fraude electrónico (sobre todo el bancario) mediante el seguimiento y control de reclamos hechos previamente por los usuarios y las posibilidades de confidencialidad debido a la alta sensibilidad de la información manejada por los diversos entes de una empresa. Por su parte, Escandón (2005) determinó la necesidad de mejorar los procesos con los que se llevan a cabo las solicitudes de equipos informáticos, tomando en cuenta la gran importancia de agilizar todos los procesos previamente descritos con la intención de mejorar la operatividad de toda organización.

Cuando se diseña un sistema debe planificarse, sustituirse o perfeccionarse el anterior lo que implica la clasificación e interpretación de los hechos, diagnóstico de problemas empleo de información y recomendación de mejoras en el sistema 
(Jacobson, Booch y Rumbaugh, 2000; Senn, 1998). Además, se deben examinar las bases de datos; construir los diagramas de ese flujo de datos respondiendo a cómo será la documentación del sistema y elaborar el diccionario de toda la información utilizada (Kendall y Kendall, 2005).

Se utilizó la Metodología RUP, un proceso complejo de ingeniería de software que provee un enfoque para asignar labores y compromisos dentro de una estructura de desarrollo. Su objetivo es asegurar la producción de software de alta calidad que satisfaga la necesidad del usuario final dentro de un tiempo y presupuesto previsible (Cardador, 2014). Tal como lo expresan Jakobson, Booch y Rumbaugh (2000) es una metodología de desarrollo iterativo enfocada hacia los casos de uso, manejo de riesgos y el manejo de la arquitectura. Las fases de inicio, elaboración, construcción y transición permiten el modelamiento, el desarrollo de bases, la aplicación de pruebas y la garantía de entrega del producto, respectivamente.

La ingeniería de software, finalmente, debe tener en cuenta ciertas bases legales sobre los delitos contra los sistemas que utilizan tecnologías. En la mayoría de los países existen disposiciones sobre la autorización a información, daño a los sistemas, la impericia o inobservancia de normas, la falsificación, el delito contra la propiedad y la privacidad de las personas, además de las multas y sanciones, civiles y penales que se pueden recibir.

\section{MÉTODO}

Con el propósito de alcanzar las mejoras señaladas se siguieron una serie de pasos para completar el diseño de un sistema automatizado de soporte técnico. De acuerdo con la naturaleza y características de problema objeto de estudio, esta investigación se enmarca dentro del nivel explicativo, en donde se analizaron las variables y los resultados se expresaron mediante hechos verificables.

El diseño utilizado es el del Proyecto Factible que consiste en "la investigación, elaboración y desarrollo de una propuesta o un modelo operativo viable para solucionar problemas, requerimientos o necesidades de organizaciones o grupos sociales; puede referirse a la formulación de políticas, programas, tecnologías, métodos o procesos" (UPEL, 2016, p. 16). El apoyo fue de tipo documental y de campo, debido a que se utilizaron los materiales para revisar la conceptualización, teorías y prácticas, por una parte, y, por la otra, se obtuvieron datos primarios en el de campo en forma directa de la realidad de la empresa.

La población estuvo conformada por 80 hombres y 100 mujeres (180 personas), entre los usuarios finales. En cuanto al personal de Área de Análisis de Soporte estaban comprendidos por 5 hombres y 5 mujeres ( 10 personas). La muestra de los usuarios finales, debido a la homogeneización del universo, se tomó al azar según la técnica de Arkin y Colton (1995), 10\% de esa población: 8 hombres y 10 mujeres. La muestra de personal del Área de Análisis de Soporte estuvo constituida por toda la población finita e intencional, es decir todo el universo: los 5 hombres y las 5 mujeres que trabajan en el área.

Las técnicas de recolección de información fueron la encuesta, la observación y el análisis de documentos (UPEL, 2016). Los instrumentos para el levantamiento de información fueron dos cuestionarios cerrados que se aplicaron tanto a los usuarios finales como a los integrantes 
del departamento de Sistemas de Información, de forma separada y con diferentes preguntas cerradas. A través de la aplicación de esta herramienta, se logró obtener la información necesaria para la factibilidad y el desarrollo del nuevo sistema libre de las carencias del sistema antiguo usado en el área de Análisis de Soporte.

La validez y confiabilidad del estudio de estableció mediante la evaluación del cuestionario por juicio de cinco expertos y se aplicó a una muestra de 10 personas con características similares, lo que arrojó una validez de 0,92. No fue necesario utilizar un grupo control debido a que la probabilidad de trabajar con usuarios y personal comporta pocas probabilidades de ausencias. Además, cada ítem se adecuó a la operacionalización de las variables (Tabla 1).

\section{Tabla 1. Conceptualización de Variables}

Objetivos
Analizar los procesos y registros que se
llevan a cabo en el sistema actual, para
identificar sus interacciones, los riesgos
y requerimientos básicos y detallados.

Establecer la fundación arquitectónica del proyecto, así como su plan de elaboración basándose en los requerimientos planteados

Proveer una base para la eliminación de riesgos potenciales de carácter técnico para el proyecto mediante la construcción de una solución preliminar en forma de prototipo basándose en la arquitectura establecida

Completar la funcionalidad del sistema mediante el estudio de los requisitos pendientes, administrando los cambios de acuerdo a las evaluaciones realizados por los usuarios.

Asegurar que el software esté disponible para los usuarios finales, eliminando los errores y defectos encontrados en las pruebas de aceptación, asegurando capacitación a los usuarios así como el soporte técnico

Variable

Procesos y registros en el área de Análisis de Soporte del Departamento de Información.

\section{Fundación} arquitectónica del proyecto

Requerimientos planteados para su desarrollo

Solución preliminar en forma de prototipo

Requisitos posteriores con base en evaluaciones

Pruebas y correcciones necesarias al sistema.

\section{Definición}

Procesos llevados a cabo para la recolección de datos de los requerimientos hechos por los usuarios $y$ su posterior documentación pre y post resolución

Desarrollo de la estructura básica del proyecto comenzando por el planteamiento de su infraestructura.

Requerimientos como punto de partida para la elaboración del proyecto, que servirán como guía para la incorporación de la infraestructura y lógica necesaria para el proyecto

Diseño preliminar de una aplicación que pueda servir como base de prueba de funcionalidad del proyecto.

Todos aquellos requerimientos basados en las pruebas llevadas a cabo en el diseño del prototipo preliminar, lo que ofrece la manera de eliminar cualquier otro riesgo potencial para el proyecto y disminuir o anular sus debilidades.

Técnicas informáticas para comprobar la calidad del sistema definitivo

Fuente: Elaboración propia. 
En la fase de análisis se ordenaron los datos obtenidos de la fase de recopilación, para luego poder sacar conclusiones y determinar la situación de estudio. Según Sabino (2000), esa masa de datos, por si sola, no nos dirá en un principio nada, no nos permitirá alcanzar ninguna conclusión si, previamente, no ejercemos sobre ella una serie de actividades tendientes a organizarla, estas acciones son las que integran el llamado procesamiento de los datos. (p. 172)

Se revisaron al detalle y sistemáticamente, se analizaron y decantaron hasta determinar aquellos que fueran útiles para la investigación y el montaje del nuevo sistema. El siguiente paso fue tabular los datos, agrupándolos y construyendo tablas, concentrados y contabilizados para la construcción de un panorama general de la realidad (Hernández, Fernández y Baptista, 2006).

Finalmente, a partir del estudio y de la conceptualización se propuso el nuevo
Sistema con sus objetivos, instrumentación e implantación dentro de la estructura de la empresa.

Previo a la fase de resultados, es necesario revisar las variables (Tabla 1) y su definición que depende de la dinámica de la construcción del proyecto, así como los interrogantes del cuestionario para el personal y los usuarios finales ofrecidos en los resultados.

\section{RESULTADOS}

Se procedió a analizar y graficar cada una de las respuestas obtenidas a través de la aplicación de los cuestionarios. En el primero, para agilizar las actividades del área de Análisis de Soporte el cuestionario se centraba en los procedimientos llevados a cabo para el registro, control, búsqueda y actualización de requerimientos de soporte técnico. A continuación se presenta el cuerpo del cuestionario y los resultados obtenidos (Tabla 2).

Tabla 2. Cuestionario para el personal

Instrucciones: Lea cuidadosamente cada una de las preguntas presentadas en este folio antes de responder, marque su opción con una $X$.

Pregunta

sí NO

1. El sistema manual utilizado para el registro y control de incidencias reportadas por los usuarios finales de equipos informáticos cumple con los estándares de manejo de información

2. La celeridad con que se realiza el registro y búsqueda de información referente a requerimientos de soporte técnico es la apropiada

3. El proceso que se lleva a cabo actualmente para el registro y búsqueda de información de incidencias funciona de forma eficiente basándose en los estándares del manejo del negocio

4. El manejo de la información referente a las incidencias reportadas por los usuarios es de alta calidad en lo que se refiere a la identificación de los actores involucrados en su registro, manejo, control y actualización

5. El procedimiento actual de registro, búsqueda y actualización de incidencias que se realiza en el área de Análisis de Soporte cuenta con copias de seguridad en caso de extravió de información

6. Cree usted que deban implantarse medidas de seguridad para el acceso a información referente a incidencias previamente reportadas por los usuarios finales

7. De implementarse un sistema automatizado para el registro, gestión y control de incidencias a nivel de soporte técnico para el área de Análisis de Soporte, cree usted que se agilizarían lo procesos antes mencionados

Fuente: elaboración propia. 
La primera pregunta sobre el sistema manual arroja un resultado de 8 personas que marcaron NO (80\%) y 2 marcaron sí (20\%); la pregunta 2, sobre la celeridad con que se realiza la búsqueda de información, 1 persona (10\%) respondió que sí es la apropiada y 9 (90\%) respondieron que NO; la pregunta 3, sobre el proceso para el registro y búsqueda de información de incidencias 10 personas (100\%) marcaron que NO funcionaba de forma eficiente; la pregunta 4 referente a si las incidencias reportadas por los usuarios es de alta calidad 9 (90\%) respondieron que $\mathrm{NO}$ y 1 (10\%) respondió que Sí; a la pregunta 5 sobre las copias de seguridad en caso de extravió de información, el 100\% (10 personas) respondieron que NO.

Las preguntas 6 y 7 se dirigían hacia las posibilidades de cambio por lo que su redacción se dirigía a las necesidades de los encuestados. Es así que a la pregunta sobre si debían implantarse medidas de seguridad para el acceso a información referente a incidencias previamente reportadas por los usuarios finales, las 10 personas (100\%) respondieron que Sí. La pregunta 7 , sobre la urgencia de implementarse un sistema automatizado para el registro, gestión y control de incidencias a nivel de soporte técnico para el área de Análisis de Soporte, recibió un 90\% (9) de respuestas Sí y una persona (10\%) marcó que NO.

En total se puede observar que las respuestas a los ítems del 1 al 5, entre 80 y $100 \%$ estuvieron en desacuerdo (marcaron $\mathrm{NO}$ ) con la eficiencia, eficacia y solvencia del sistema manual, mientras que para los ítems 6 y 7 , sobre las necesidades de actualización y cambio entre el 90 y $100 \%$ estuvieron de acuerdo (marcaron Sí). Con respecto al cuestionario para los Usuarios Finales (Tabla 3) la presentación y los resultados fueron los siguientes:

Tabla 3. Cuestionario para usuarios finales

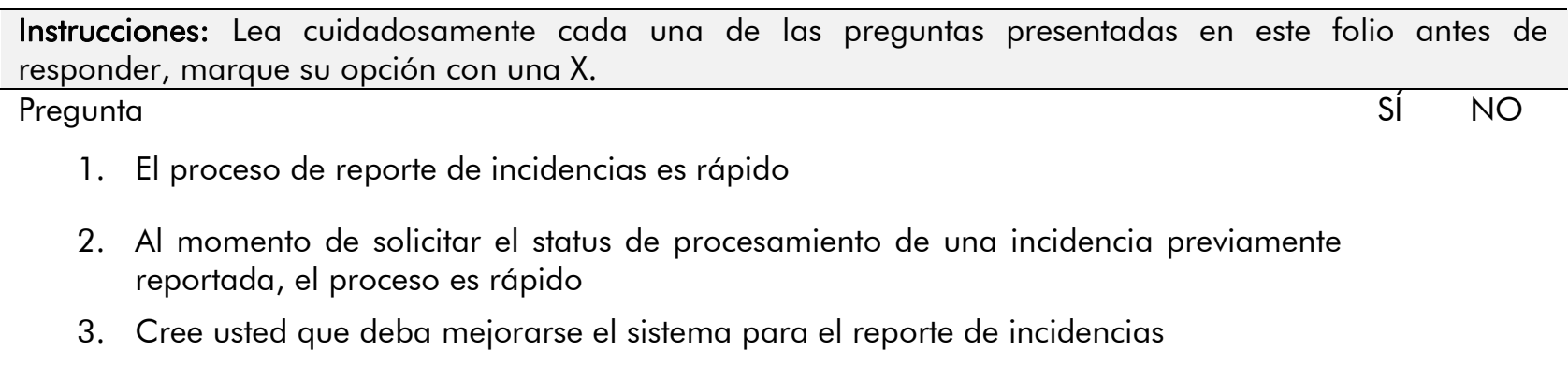

Fuente: elaboración propia

El total de la muestra tomada era de 18 personas que equivalen al $100 \%$; para la pregunta 1, tres (3) personas (17\%) respondieron que el proceso de reporte de incidencias sí es rápido y 15 (83\%) respondieron que $\mathrm{No}$; en la pregunta dos 16 personas $(89 \%)$ respondieron que al momento de solicitar el status de procesamiento de una incidencia, el proceso NO es rápido, y 2 personas (11\%) respondieron que Sí. Sobre la pregunta 3, las 18 personas $(100 \%)$ respondieron que sí debía mejorarse el reporte de incidencias. 


\section{Diagnóstico de la situación que debe ser mejorada}

Los resultados mostraron que, debido a la carencia de políticas de seguridad, a nivel informático, en cuanto al manejo de data referente a requerimientos de soporte técnico, se perdían o extraviaba (incluso intencionalmente) información referente a los incidentes en marcha, que interrumpían la continuidad del negocio. El manejo manual de todos los procedimientos de registro y almacenamiento de la data representó tardanza del procesamiento de solicitudes de soporte técnico, a causa de la inexactitud en los criterios. Un factor extra que ralentizaba la resolución de incidentes reportados, estuvo representado en la ausencia de una fuente de conocimientos que asociara casos previamente reportados con métodos de solución ya aplicados. En el momento de afrontar nuevas incidencias o problemáticas, no se contaba con una base de datos sólida que pudiera ofrecer pistas de cómo se llevan a cabo la eliminación de cualquiera de las posibles fallas en cualquiera de los sistemas que manejan información.

Igualmente, se pudo diagnosticar la falta de métodos de organización efectivos una vez que se llevaba a cabo el registro de un incidente, debido a que toda la información reportada era manejada por una sola persona asignada a la recepción de los casos. No existía ningún mecanismo de recepción de incidentes que permitiera el manejo centralizado de la información para proveer continuidad en las operaciones que involucraban el soporte técnico. Del mismo modo, los procedimientos de actualización de información de incidentes eran prácticamente inexistentes ya que no se contaba con una manera de trabajar con este tipo de información apropiadamente.
Como consecuencia, la atención de los requerimientos efectuados por los usuarios se veía comprometida y, por añadidura, la continuidad del negocio de la empresa.

Luego del diagnóstico, la alternativa más confiable, económica y eficiente fue desarrollar el sistema dentro de la misma institución, con las herramientas de software disponible para ese momento a fin de reducir los costos de inversión. Los criterios de evaluación para llegar a esta conclusión se basaron en (1) costos implicados en el desarrollo de la herramienta; (2) tiempo requerido para completar la tarea de análisis y desarrollo de la herramienta; (3) calidad y disponibilidad de soporte para la herramienta y para su desarrollo y expansión.

En la empresa se contaba con personal autorizado para manejo de la información que circularía a través del sistema, capacidad de actualización y desarrollo de nuevos módulos obteniendo la información de requerimientos de primera mano dentro de la empresa y no representarían un costo adicional excesivo para su desarrollo. Además, se contaba con personal capacitado dentro de la empresa para el desarrollo del producto.

\section{Estudio de las factibilidades}

En un estudio de factibilidad como este una de las primeras exploraciones se refirió a la factibilidad técnica. Con respecto al hardware se resolvieron satisfactoriamente por la empresa mediante la adquisición de un lote de equipos de última generación (laptops y desktops Lenovo para el manejo de la herramienta dentro de las instalaciones de la empresa y PDA HP Glisten para aquellos asignados fuera de la empresa). Además, se requirieron equipos dedicados 
para el desarrollo y manejo de la herramienta: 2 Servidores HP ProLiant ML350, 1 Rack servidores 3 slots y 6 Computadores Lenovo †400. (En la actualidad podrían conmutarse con virtualización los 2 servidores por 1 Rack de 1 solo slot). Igualmente, a la fecha podría hacerse con 1 servidor proliant, Citrix Xenserver como hipervisor, 2 ó 3 máquinas virtuales que van a operar encima del hipervisor (con Linux o licencias Windows server).

En el caso del software, las aplicaciones, el desarrollo e incorporación de la base de datos serían más convenientes a través de un entorno orientado hacia el ámbito Web y el desarrollo de una base de datos para el manejo y uso de la información. En este caso, MySQL Enterprise Edition.

Con respecto a los recursos humanos la empresa ya contaba con personal capacitado para el desarrollo de la herramienta. Sin embargo, fue necesaria la incorporación de ciertos roles como de líderes y analistas para equipos y operaciones dedicados, exclusivamente, al desarrollo de la nueva herramienta.

En lo que atañe al balance entre costos y beneficios el peso total de los gastos previstos frente al total de los beneficios fue, efectivamente, la mejor opción y la más rentable. Los costos fueron temporales y los beneficios fueron permanentes y a largo plazo, de índole social, económica, calidad, servicio (tangibles $O$ intangibles), que sirvieron de base para su incorporación a sistemas más modernos en comparación con otras empresas del ramo.

Sobre la factibilidad operativa no fue necesaria la adquisición de licencias o de programas externos, ya que la empresa contaba -como la mayoría de su ramo y envergadura- con las licencias. En el caso de la implantación de Linux, este no requiere pago adicional ya que es un software "Open Source". Igualmente, se mantuvieron las normas de confidencialidad y las normativas de la aplicación y uso de los sistemas de información para el manejo de data de usuarios, así como el código de conducta para empleo de equipos informáticos que fueron aprobados mediante un proceso de auditoría legal. En cuanto a la aplicación para implantar la propuesta que solventaría el estado del sistema manual, se recibió la plena autorización legal al no infringir ninguna de las leyes correspondientes a los sistemas de información aplicadas nacional e internacionalmente.

Como en todo estudio para ofrecer soluciones el sistema dispuso de un manual de usuario impreso y en formato digital no alterable. Cada uno de los usuarios autorizados al manejo del sistema en sus diferentes módulos tuvo acceso a la documentación disponible a través de los canales regulados. Cada uno de los accesos al sistema, procesos, cálculos, consultas y otras operaciones generadas por el sistema estuvieron debidamente documentados. En cuanto al posterior entrenamiento 0 formación de usuarios para el uso del sistema, se realizó con la celeridad adecuada. De igual manera, se colocó a la disposición, para consulta del usuario, un manual de ayuda rápida en línea. Cada uno de los módulos que conformaron el sistema estuvo provisto de documentación amigable y de fácil entendimiento para el usuario, para su orientación, permanentemente, dentro de las facilidades que ofrece el sistema. 


\section{Sistema propuesto}

\section{Descripción general del sistema propuesto}

Se lleva a cabo el desarrollo del sistema propuesto, basado en los análisis de factibilidad técnica, económica y operativa previamente efectuados. Se creó la base de datos con los registros y las tablas relacionadas con las solicitudes de soporte de diversa índole, lo que permitió establecer un centro de compilación de incidencias de forma automatizada y centralizada, y al Analista de Soporte encargado de la recepción de los reclamos así como a los grupos de resolución asignados tener acceso a la información de registro y actualización de cada uno de los incidentes.

Esta base de datos ahora está disponible y alojada dentro de los ámbitos de la red interna de la empresa, así como para los usuarios remotos mediante un vínculo de conexión segura: cada usuario tiene acceso solo a los datos relacionados con las incidencias reportadas desde su perfil; los usuarios finales tienen acceso a la lectura de datos y pueden imprimir reportes en papel; únicamente el Analista de Soporte y los Grupos de resolución tienen acceso completo al sistema y a su base de datos. El sistema ha seguido actualizándose, de acuerdo con los nuevos avances a partir de su método precursor.

Todo esto se completó mediante un sistema de gestión y manejo de incidencias soportado en un entorno grafico o interfaz que permitiera la interacción entre los usuarios autorizados para su control y la base de datos con todas las incidencias reportadas. Dicho sistema de gestión y manejo de incidencias estuvo compuesto por ventanas gráficas, pantallas e instrucciones del programa. De esta forma, el usuario interactuaba con la base de datos $\sin$ acceder directamente o en un nivel que no le correspondiera.

El sistema además de herramienta de información confiable para los entes de Control de Operaciones y Rendimiento ha servido para monitorear y llevar la cuenta de la operatividad de los efectivos de ventas y de oficina. Esto agrupa el conjunto de políticas, objetivos, metas, misión y visión tanto del Departamento de Sistemas de Información como de las subunidades de la empresa lo que ha generado mejoras en la ejecución de sus tareas funcionales, procedimientos, herramientas de control y de evaluación.

El Analista de Soporte Técnico ahora dispone de mayor tiempo para el análisis de los incidentes y su delegación a los grupos de resolución sin afectar las operaciones de registro de nuevas incidencias, lo cual ha representado un valor agregado para la empresa, el Departamento de Sistemas de Información y el Área de Soporte Técnico. Esto ha constituido el establecimiento de una estructura básica o esqueleto sobre el cual se puede soportar la gestión de las incidencias, aun con los cambios en las tecnologías.

En suma se ha podido cumplir con los objetivos principales del sistema:

1. Reducir el tiempo de respuesta simplificando todas las tareas

2. Ofrecer seguridad y rapidez en el proceso de registro de incidencias

3. Incentivar un mejor uso del tiempo de los analistas mediante la delegación del registro de las incidencias en el sistema automatizado

4. Establecer una base de conocimientos centralizados y sólidos que sirva como 
marco de consulta para la resolución de futuras problemáticas. equipos informáticos mediante el estudio de su ambiente de incidencias.

5. Generar estadísticas acertadas acerca del desempeño de los usuarios de

La carta estructurada, puede observarse en la Figura 1:

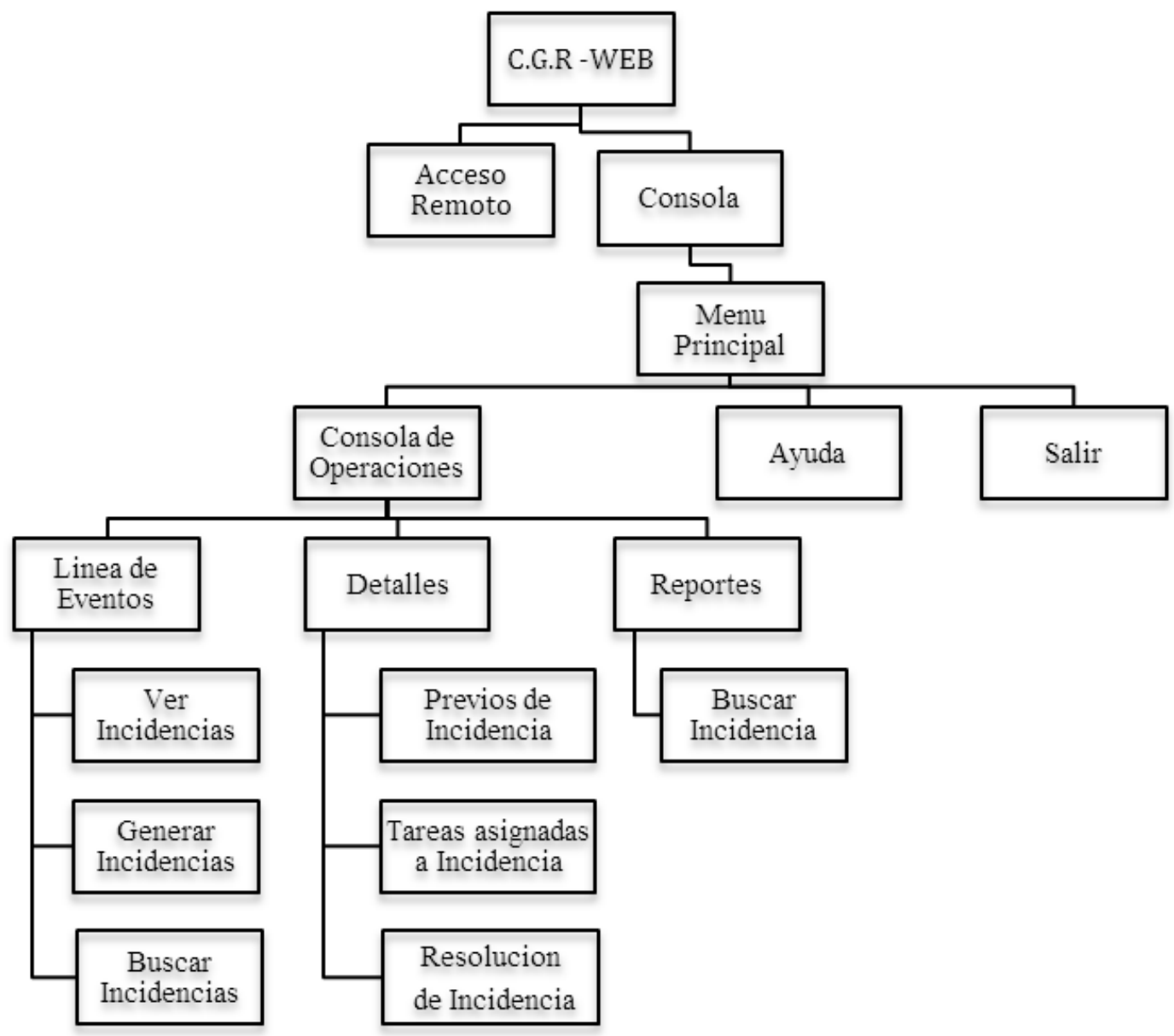

Figura 1. Carta estructurada. Fuente: Elaboración propia

También se puede observar la descripción de las entidades en la Tabla 4, que son puntos significativos en la Figura 2. 
Tabla 4. Descripción de Entidades

\section{Nombre}

Usuario Final

Operaciones

\section{Descripción}

Es la persona que se beneficia del servicio de soporte mediante la atención del requerimiento

Es el ente que se beneficia de la información acerca del status operativo a nivel informático de todos los departamentos

\section{Propósito}

Es la entidad encargada de elaborar un requerimiento de soporte

Es la entidad encargada de solicitar la información referente a las operaciones llevadas a cabo por los usuarios

Fuente: Elaboración propia 


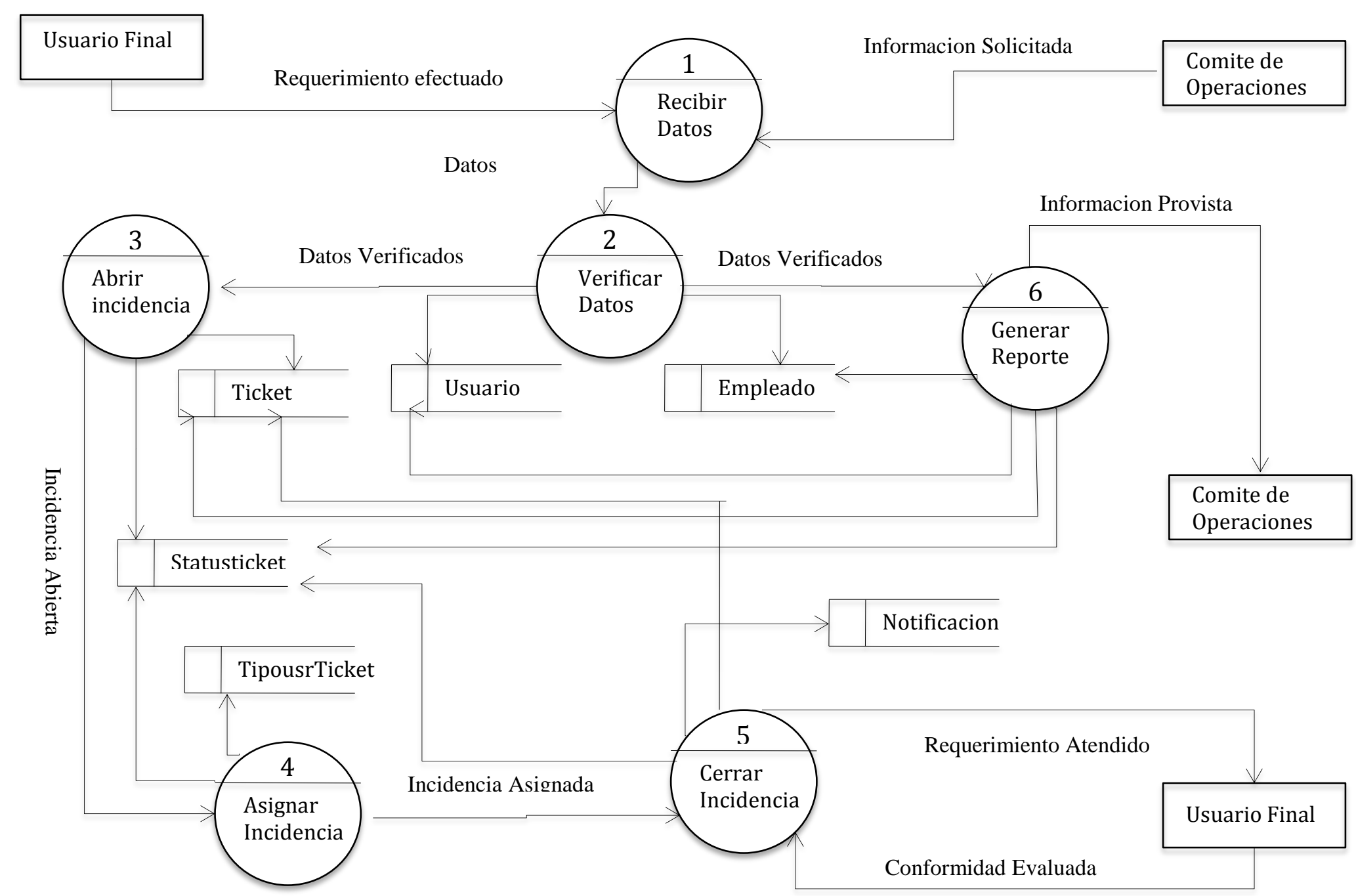

Figura 2. Diagrama de Sistema (Nivel 1) 


\section{Modelo lógico}

Si bien las bases de datos orientadas a objetos se estén volviendo populares, la base de datos relacional sigue siendo el método predominante para almacenarlos. Aunque, diversos métodos se pueden utilizar para modelar la base de datos relacional en la que el sistema está basado, los diagramas tradicionales de modelado de datos capturan más información acerca de la base de datos relacional y son más adecuados para su modelado.

La implantación del sistema trata de traducir información desde múltiples niveles a código y volcarla así en una estructura de base de datos. Cuando se modela un sistema de información de envergadura, es útil fragmentar el sistema en capas, las que incluyen los objetos de la interfaz gráfica de usuario, su capa de aplicación (objetos de implementación) y su capa de datos que incluye la estructura de base de datos y el acceso a objetos.

Tal como lo mencionan Kendall y Kendall (2005) "Las bases de datos no son tan solo una colección de archivos. Más bien, una base de datos es una fuente central de datos destinados a compartirse entre muchos usuarios para una diversidad de aplicaciones" (p. 444). Los diagramas Entidad-Relación son un lenguaje grafico para describir conceptos de forma interactiva. Informalmente, son simples dibujos o gráficos que, sabiendo interpretar, describen la información que requiere comunicar o plasmar. Son de gran utilidad porque ahorran tiempo al momento de validad representativamente una idea. El ser humano percibe y retiene más información cuando enfoca los elementos de forma visual.

Para lograr otro de los objetivos propuestos, se desarrolló el diseño de base de datos que forma parte del sistema (ver Figura 3). Esto contempla la creación de tablas y las relaciones entre sí, las cuales pasaron a través del proceso de normalización y se detallaron en los diccionarios de datos. Igualmente, se detallaron los campos requeridos para armar y almacenar los registros de bases de datos (Tabla 5). Al implementar el diseño relacional, se establece una estrategia en la que se busca marcar referencias entre el diagrama de relaciones lógico y un diagrama físico que representa el objetivo. El diagrama físico puede ser normalizado y lograr un diseño de base de datos que tiene tiempos eficientes de acceso a los datos. Las relaciones entre entidades se resuelven por las estructuras de tablas. 


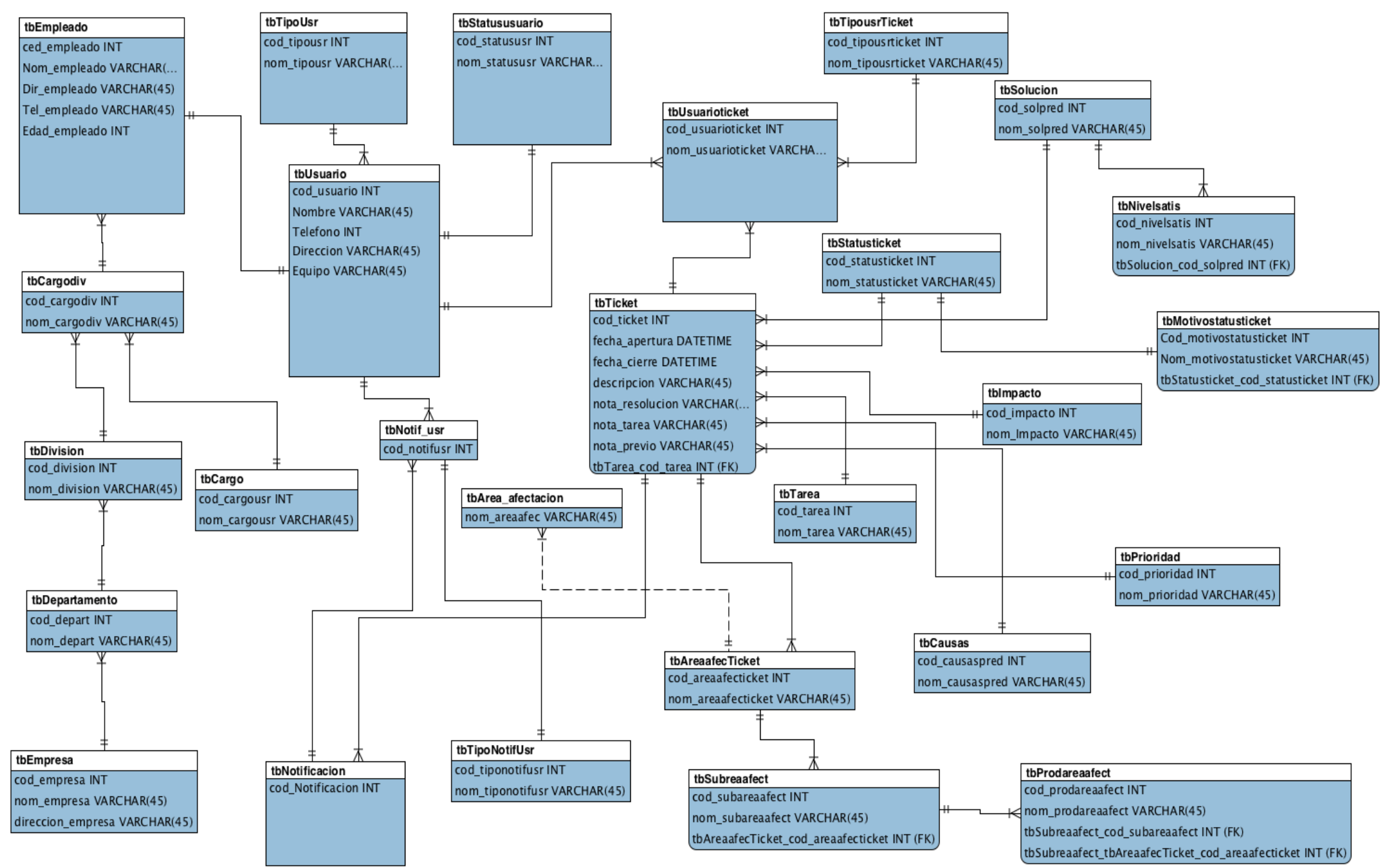

Figura 3. Diagrama Entidad-Relación. Fuente: Elaboración propia 
Tabla 5. Descripción de los Procesos. Nivel 1

\begin{tabular}{llll}
\hline Número & Nombre & Flujo de Entrada & Flujo de Salida \\
1 & Recibir Datos & - Requerimiento efectuado & - Datos Recibidos \\
2 & Verificar datos & - Datos Recibidos & - Datos Verificados \\
3 & Abrir Incidencia & - Datos Verificados & - Incidencia Abierta \\
4 & Asignar Incidencia & - Incidencia Abierta & - Incidencia Asignada \\
5 & Cerrar Incidencia & - Incidencia Asignada & - Requerimiento Atendido \\
& & & - Conformidad Evaluada \\
6 & Generar Reporte & - Datos Verificados & - Información Provista \\
\hline
\end{tabular}

Fuente: Elaboración propia

La realización de este proyecto llevó a obtener beneficios a nivel operativo, financiero y logístico: mejora de los procesos de registro y control de solicitudes de soporte técnico; disminución en los tiempos de respuesta a los usuarios ya que se disminuyó parcial o totalmente el intermediario en la recepción de la solicitud; compilación segura y constante de una base de conocimiento confiable que provee información para la corrección de fallas y mejoras en la implantación de próximos proyectos. Este proceso se observa en forma secuencial en las figuras 4,5 y 6 .

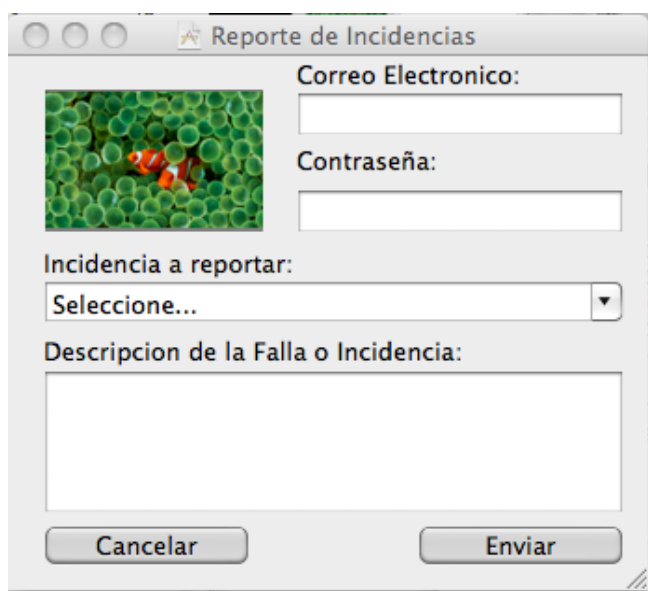

Esta es la pantalla inicial del módulo de Acceso Remoto del sistema G.R.C - Web a la cual se accesaba mediante conexión a un vínculo web

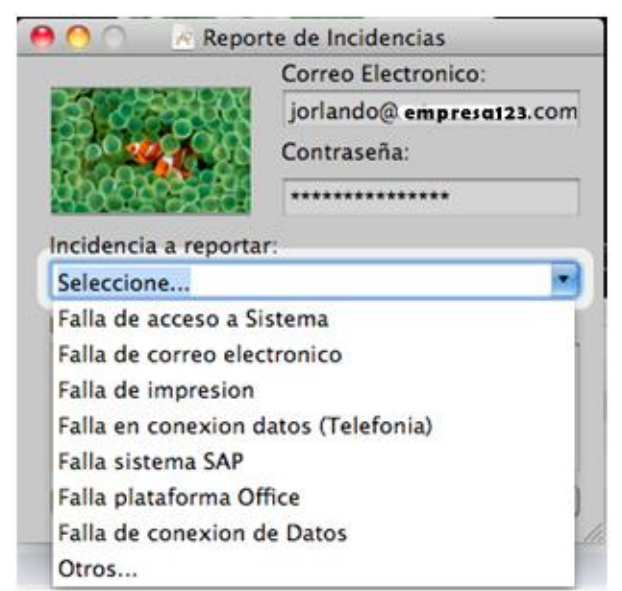

Se procede al llenado de la ventana incluyendo correo electrónico y contraseña (previamente validados por la intranet de la empresa). Adicionalmente, se selecciona alguna de las opciones de la lista desplegable que describe mejor la falla presentada al usuario.

Figura 4. Manual del Sistema. GRC - WEB / Acceso Remoto (pasos 1 y 2). Fuente: Elaboración propia 


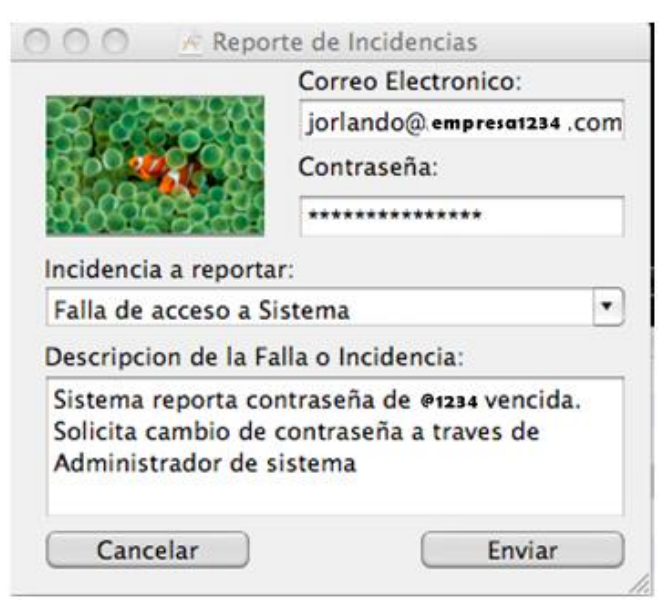

Una vez seleccionada la opción, se incluye una breve descripción de la incidencia. Luego, se presiona el botón "Enviar"

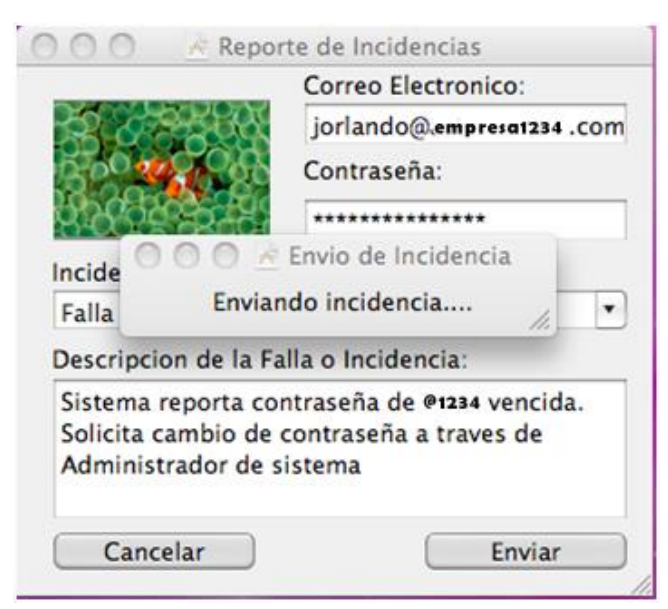

Una vez presionado el botón "Enviar", aparecerá el aviso de procesamiento del requerimiento

Figura 5. Manual del Sistema. GRC - WEB / Acceso Remoto (pasos 3 y 4). Fuente: elaboración propia

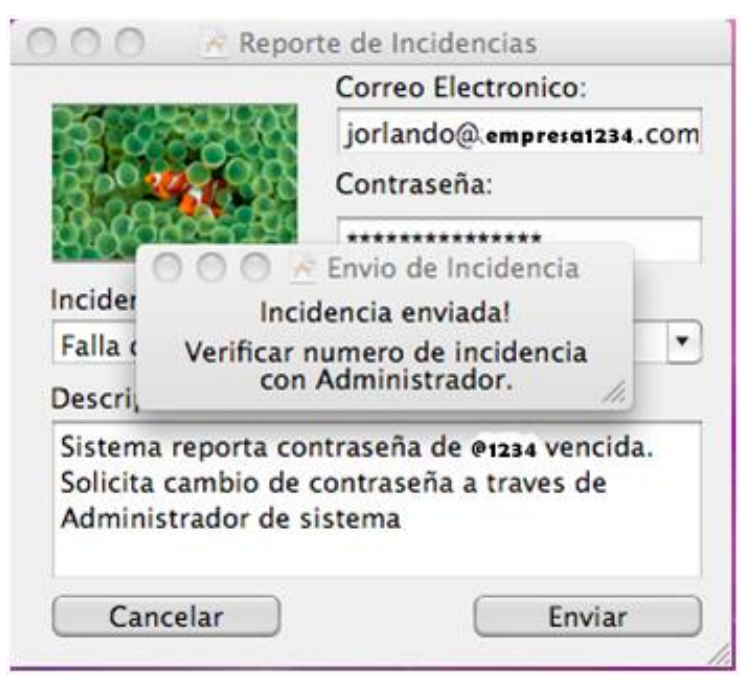

Una vez procesado el requerimiento, aparece un aviso de envío realizado previa validación del correo y la contraseña por parte del sistema.

Figura 6. Manual del Sistema. GRC - WEB / Acceso Remoto (paso 5). Fuente: elaboración propia

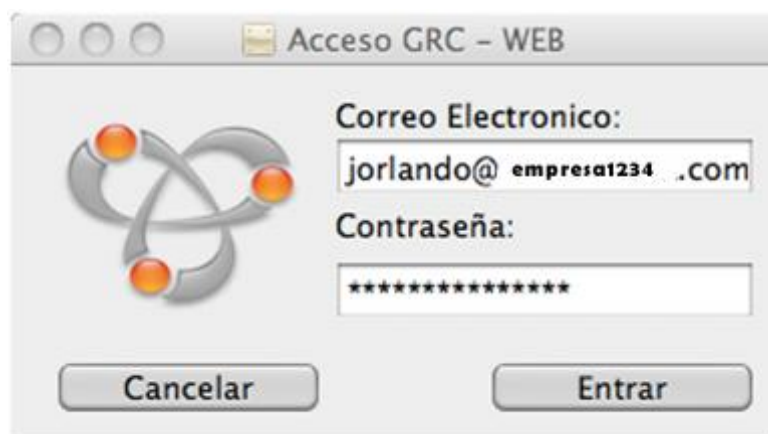

Desde el vínculo de acceso a la consola principal, el explorador web mostrará el módulo de acceso al sistema. Para poder acceder, se coloca el correo y contraseña. Luego, se presiona el botón "Entrar"

Figura 7. Manual del Sistema. GRC - WEB / Consola de Operaciones (Entrada). Fuente: elaboración propia 
Una vez dentro del sistema (ver Figura 7), se podrán ver sus 3 elementos básicos. El primer elemento es la Línea de Eventos (ver Figura 8), la cual es popular conforme los usuarios reporten sus incidencias a través del módulo remoto. Desde la Línea de Eventos se podrá tener acceso a cada uno de los tickets de forma individual.

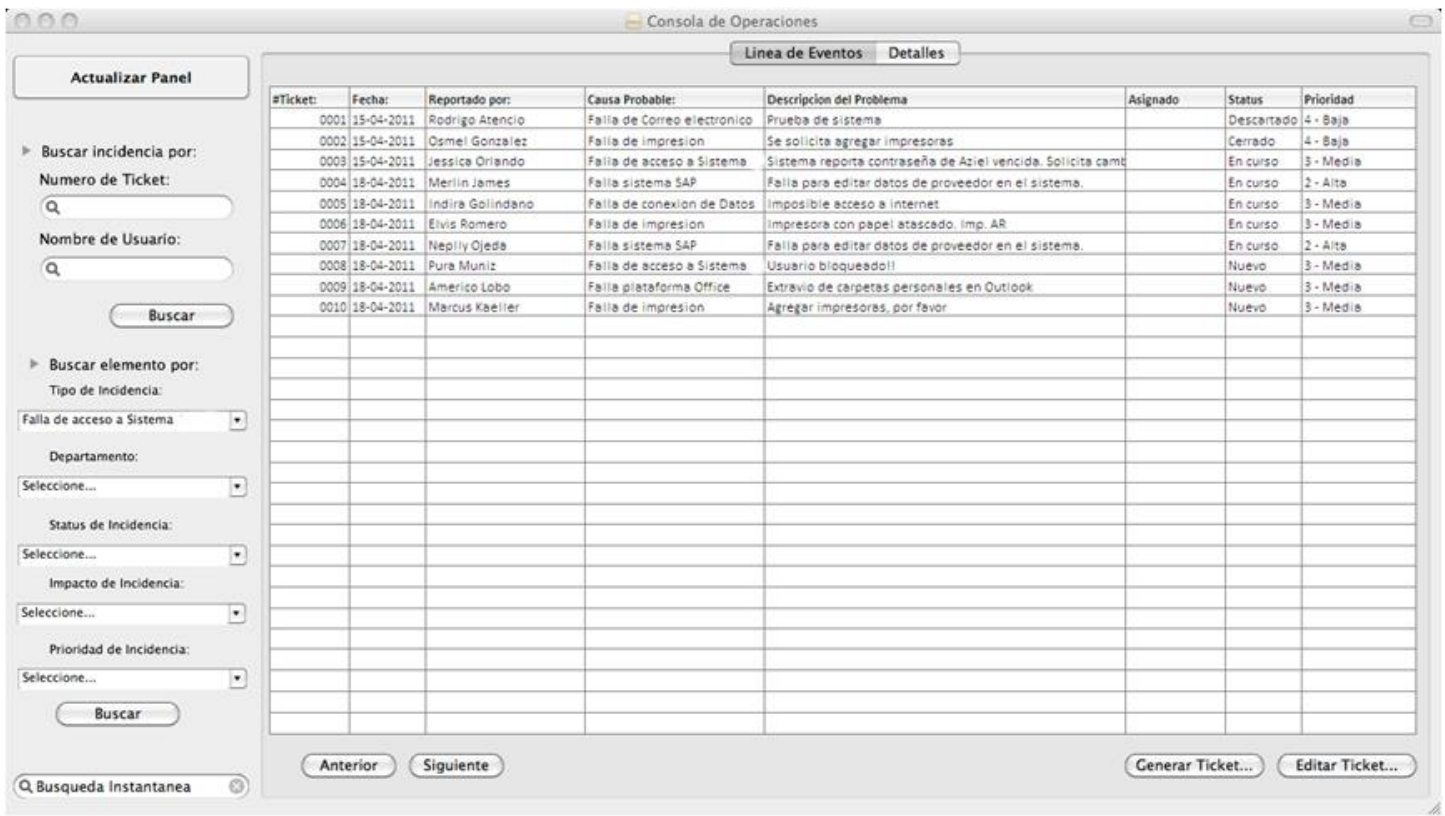

Figura 8. Manual del Sistema. GRC - WEB / Consola de Operaciones. Línea de Eventos. Fuente: elaboración propia

El segundo elementos es el panel de búsqueda ubicado en la parte izquierda (ver Figura 9) de la consola desde la cual puede efectuarse cualquier tipo de búsqueda de forma rápida. Como ejemplo, tenemos la búsqueda de un ticket de requerimiento generado en base a una incidencia reportada por un usuario (Tabla 6).

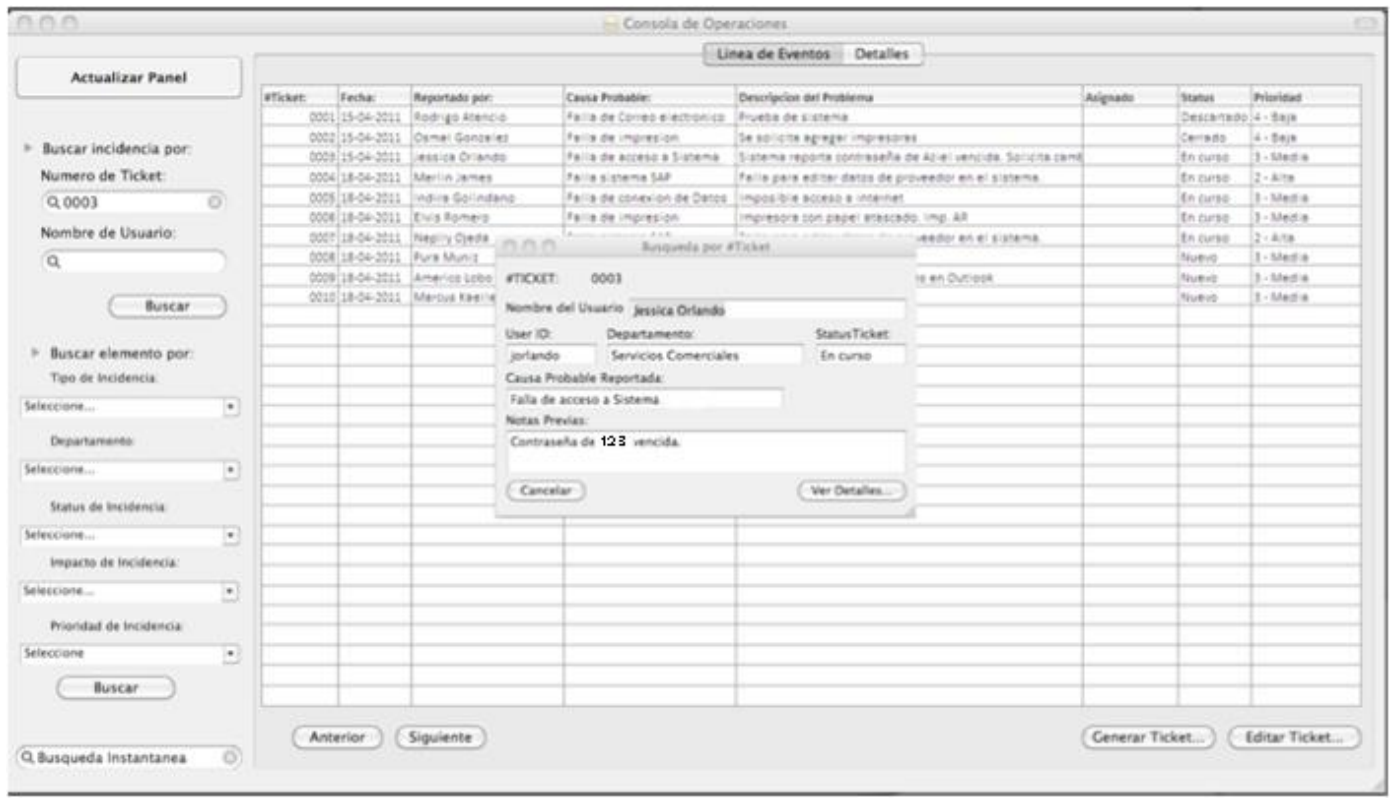

Figura 9. Manual del Sistema. GRC - WEB / Consola de Operaciones. Búsqueda. Fuente: elaboración propia 
El tercer elemento del sistema es la ventana "Detalles" (ver Figura 10). Desde allí se ven y editan todos los detalles de los tickets generados con base en las incidencias. Desde la pestaña Previos se generan tickets y toman notas de la incidencia y la información básica del ticket está siempre visible, facilitando la labor del Analista de Soporte. Desde la pestaña Tareas, se puede agregar la categorización de la incidencia y crearse tareas predeterminadas para la resolución de incidencias de forma que estén disponibles de forma rápida.

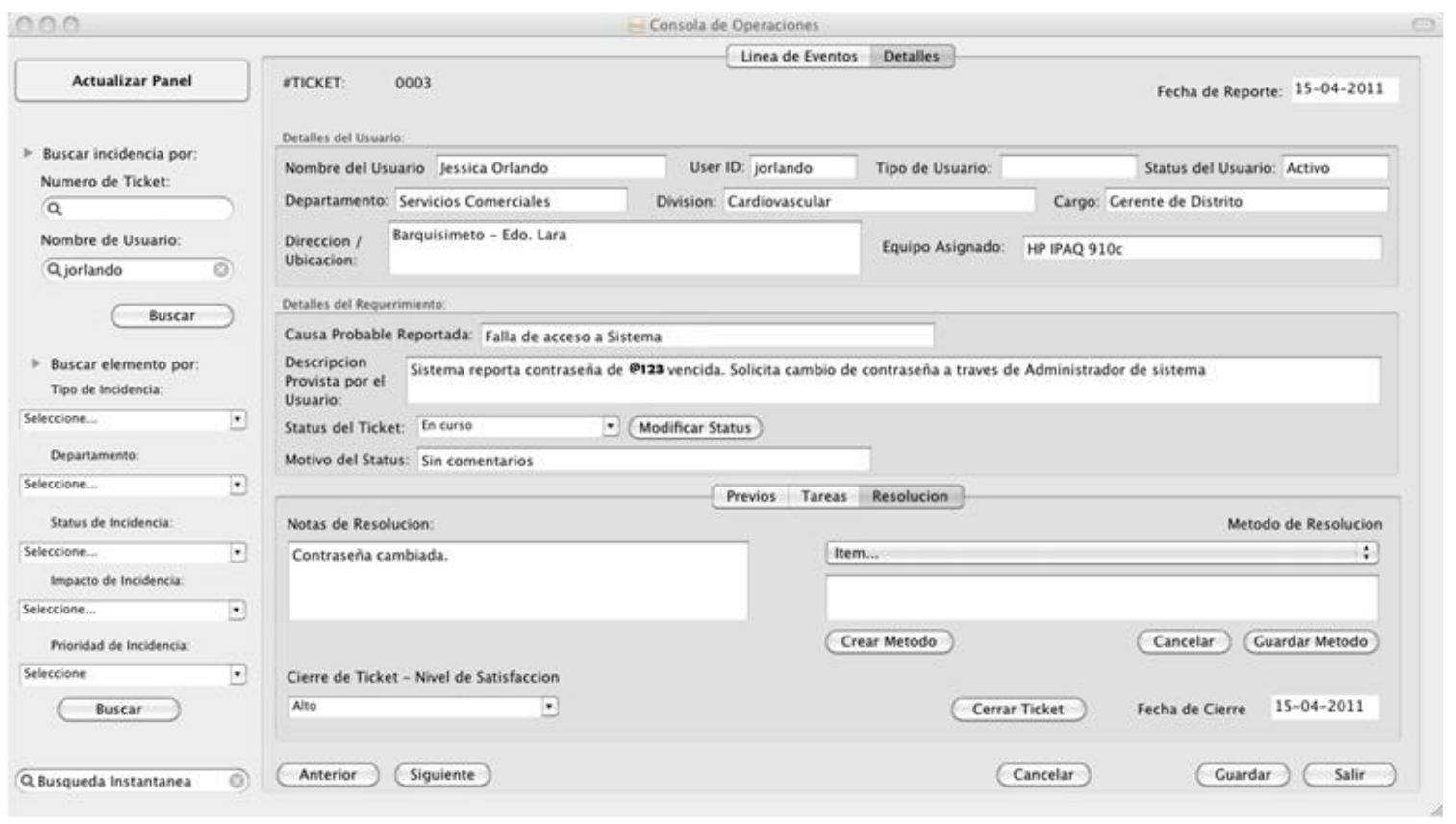

Figura 10. Manual del Sistema. GRC - WEB / Consola de operaciones. Detalles. Fuente: elaboración propia

Desde la pestaña tareas se puede agregar información y notas acerca de la resolución de las incidencias predeterminando métodos de solución para cierto tipo de incidencia. 
Tabla 6. Caso de Uso. Reporte de incidencia a través de Acceso Remoto

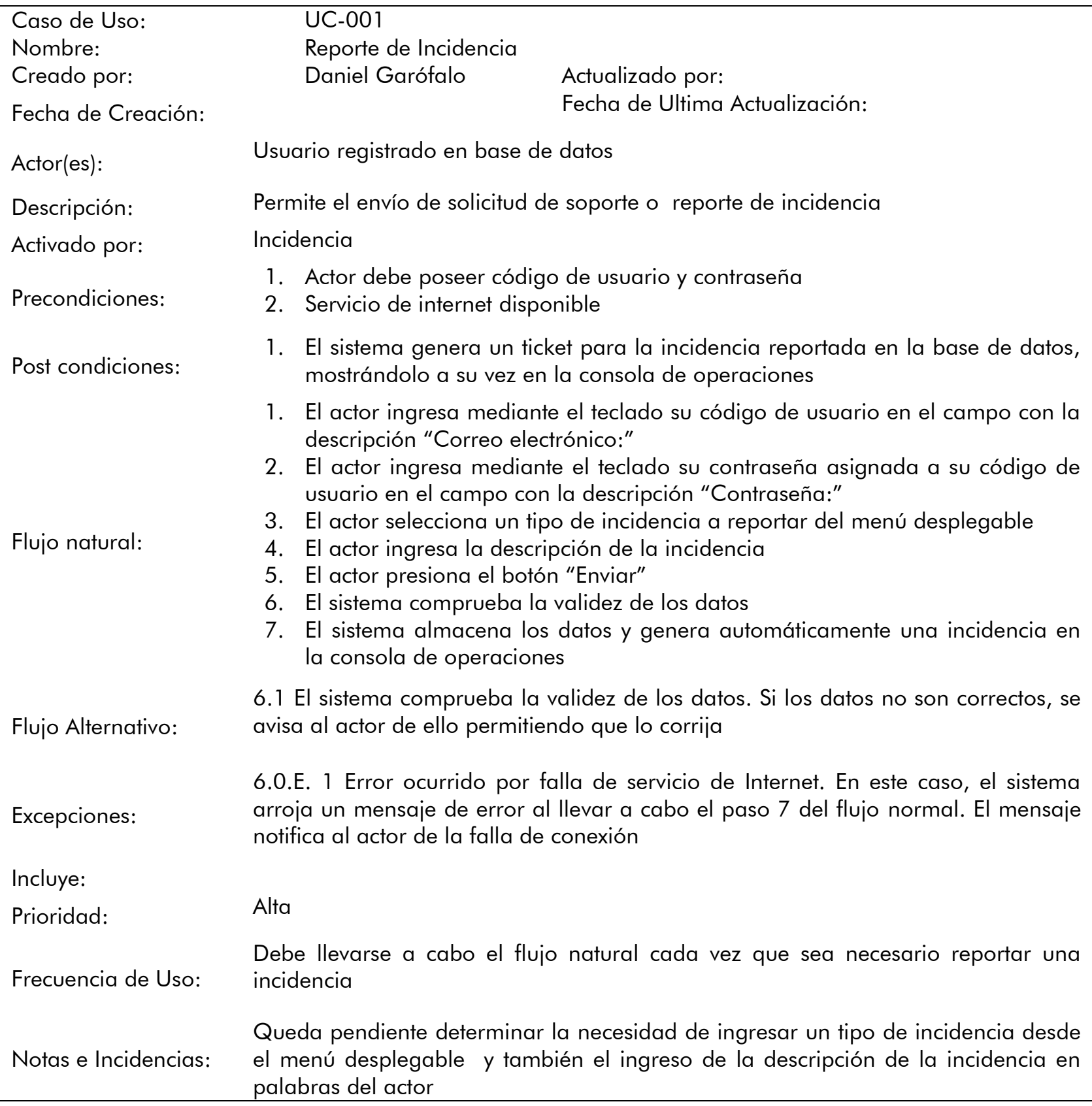

Fuente: elaboración propia

CONCLUSIONES

El proyecto estuvo orientado a la elaboración de un sistema de información automatizado y fue diseñado y construido para ser utilizado en el área de Análisis de Soporte Técnico de empresas de alta rotación de usuarios en el área farmacéutica. En el estudio se recopiló abundante información referente al manejo del sistema manual para la gestión, registro y control de incidencias reportadas por usuarios finales de equipos informáticos. Luego del levantamiento de información se llevó a cabo su análisis, para determinar los problemas y fallas existentes en cuanto a organización y seguridad de los datos y los requerimientos de los usuarios de un nuevo método que permitiese el mejor manejo de la información dentro del área de Análisis de Soporte Técnico y las deficiencias del sistema 
manual anterior. Los resultados del análisis de estos datos, arrojaron que existía la necesidad de crear un nuevo sistema para la optimización del manejo y la seguridad de los datos que se manipulan.

Con la codificación de los datos recolectados se procedió a diseñar un sistema automatizado que pudiera optimizar y dar rapidez al manejo de los datos en el área de Análisis de Soporte del departamento de Sistemas de Información, y que al mismo tiempo, brindara seguridad y respaldo. Además, con el uso de este sistema ahora no solo se agilizó el manejo de los datos, sino que se evitaron retrasos y contratiempos que involucraban a los demás entes que también juegan un papel clave en el desempeño de las actividades de la empresa, como los son los proveedores, clientes, médicos, centros asistenciales y otros.

Se diseñó y construyó el sistema automatizado bajo ambiente web para la Gestión y Control de Requerimientos de soporte técnico, con este sistema se logró optimizar los procesos de registro y consulta de los datos, reduciendo significativamente el tiempo empleado en estas actividades y ofreciendo seguridad a la información al mismo tiempo de crear una apertura para continuar en la modernización y ser ejemplo para otras empresas. Además se incluyó un manual de uso y recomendaciones para el mantenimiento del sistema instalado con un sector de respaldo. De esta manera, el sistema podrá ser recuperado en caso de anomalías en su ejecución o daño de hardware. Con el cumplimiento de todos los objetivos se llevó a la empresa a una nueva etapa que ha continuado perfeccionándose en el curso de los años.

\section{REFERENCIAS}

Alcalde, E. (2001). Informática Básica. 2da Edición. México: Editorial McGraw-Hill

Arkin, H. y Colton. R. (1995). Métodos estadísticos. Serie Compendios Científicos. México: CECSA

Cardador, A. (2014). Implantación de aplicaciones web en entornos internet, intranet y extranet. IFCD0210. Málaga, España: IC Editorial

Escandón, R. (2005). Implementación de un sistema Web para la solicitud de préstamos de equipos al personal de Funvisis desarrollado con software libre. (Trabajo de Grado), Universidad Alejandro de Humboldt, Caracas, Venezuela

Estévez, M. (2008). Desarrollo y Aplicación de un sistema Helpdesk para el control de servicios de Recursos Humanos del INCE Miranda. (Trabajo de Grado), Universidad Alejandro de Humboldt, Caracas, Venezuela

Hamidian, B. y Ospino, G. (2015) ¿̇Por qué los sistemas de información son esenciales? ANUARIO. 38, 161-183. Recuperado de http://servicio.bc.uc.edu.ve/derecho/rev ista/idc38/art07.pdf

Hernández, R., Fernández, C. y Baptista. P. (2006). Metodología de la Investigación. México: McGraw-Hill Interamericana

Howard, P. (2003). Information Security Management. Handbook. New York, EEUU: Tipton \& Krause

Jacobson, I., Booch, G. y Rumbaugh, J. (2000). El Proceso Unificado de Desarrollo de Software. Madrid, España: Ed. Addison Wesley

Kendall, K. y Kendall, J. (2005). Análisis y diseño de sistemas. 6ta. Edición. México: Pearson Educación

Sabino, C. (2000). El proceso de investigación: una introducción teóricopráctica. Caracas, Venezuela: Editorial Panapo 
Senn, J. (1998). Análisis y Diseño de Sistemas de Información. 2da Edición. México: Editorial McGraw - Hill

Stoner, J., Freeman, R. y Gilbert, D. (1996). Administración. México.: Prentice Hall Hispanoamericana

UPEL. (2006). Manual de Trabajos de Grado de Especialización y Maestría y Tesis Doctorales. Caracas: FEDUPEL
Uzcátegui, L. (2006). Diseño de un sistema de control, gestión y seguimiento de reclamos en tarietas de débito, para la unidad de prevención de fraude del Banco Mercantil C.A. (Trabajo de Grado), Universidad Alejandro de Humboldt, Caracas, Venezuela 